\section{Revisões sistemáticas de antibioticoprofilaxia em cesarianas}

\author{
Systematic reviews of antibiotic prophylaxis \\ in cesareans
}

\footnotetext{
1 Agência Nacional de Saúde Suplementar, Rio de Janeiro, Brasil. 2 Escola Nacional de Saúde Pública Sergio Arouca, Fundação Oswaldo Cruz, Rio de Janeiro, Brasil.

Correspondência L. Krauss-Silva Departamento de Administração e Planejamento em Saúde, Escola Nacional de Saúde Pública Sergio Arouca, Fundação Oswaldo Cruz. Rua Leopoldo Bulhões 1480, Rio de Janeiro, $R J$ 21041-210, Brasil. leticiak@ensp.fiocruz.br
}

\begin{abstract}
This study reviews the available evidence on the efficacy of antibiotic prophylaxis in cesarean sections. The study included a detailed analysis of the quality of design and performance and the clinical heterogeneity of selected clinical trials. Meta-analysis of placebo-controlled trials estimated an efficacy of some 65\% for the two study endpoints, endometritis and surgical wound infection, corresponding to a decrease in infection rates of some $11 \%$ and 5\%, respectively. Sensitivity analyses showed summary effects similar to those observed for all studies. Results for the non-elective cesarean sections subgroup (12 studies) indicated a relevant benefit for both endpoints, corresponding to a decrease in incidence rates of some 14\% (endometritis) and 5\% (surgical infection wound). For elective surgeries (two trials), no relevant benefit was found. Evidence from comparative trials on number of doses was limited due to important methodological shortcomings and to the small number of patients enrolled (three trials). Comparative drug trials (seven studies) did not show evidence of different performance between first and second-generation cephalosporins.
\end{abstract}

Cesarean Section; Antibiotic Prophylaxis; Review Literature
Ana Cristina Marques Martins 1

Letícia Krauss-Silva 2

\section{Introdução}

As cesarianas podem implicar infecções póscirúrgicas mais ou menos graves, desde feridas operatórias passando por endometrite até, mais raramente, sepsis. Os microorganismos associados à infecção pós-cesariana são diversos, sendo oriundos da pele e da invasão ascendente do trato genital, durante a gestação e o préparto ${ }^{1}$. Mais recentemente, métodos de biologia molecular têm ajudado a ampliar o conhecimento sobre o processo e os tipos de microrganismos associados à invasão ascendente 2 .

Uma das formas de diminuir a incidência de infecção pós-cesariana é o tratamento da vaginose bacteriana sintomática e assintomática com antibióticos durante o pré-natal 3 , entretanto, boa parte das pacientes reverte espontaneamente essa condição antes do parto 4 . Outra alternativa tem sido o uso de antibioticoprofilaxia durante a cirurgia, após o clampeamento do cordão umbilical.

As taxas de infecção pós-cesariana, sem a antibioticoprofilaxia, relatadas na literatura, vêm diminuindo, nas últimas décadas, de $60 \%$ para cerca de $18 \% 5$. Essa queda está provavelmente relacionada à implementação de outras medidas anti-infecção nos processos de assepsia e esterilização, a melhorias nos materiais utilizados e ao aperfeiçoamento das técnicas cirúrgicas; no entanto, as taxas ainda se mantêm em níveis altos 5 .

Embora não tenhamos encontrado dados recentes sobre a incidência de infecção pós-cirúr- 
gica em cesarianas para o Brasil, estima-se que esse seja um problema relevante no país, inclusive pelas altas taxas desse tipo de cirurgia registradas em nosso meio. No ano de 2002, segundo dados extraídos do Sistema de Informações de Nascidos Vivos (SINASC), o percentual de cesarianas no Brasil foi de $38,6 \%$, o que equivale a cerca de 1.200.000 partos cirúrgicos.

De acordo com o Consenso sobre o Uso Racional de Antimicrobianos do Ministério da Saúde 6, tais drogas podem prevenir a ocorrência de infecção pós-cesariana. O documento, todavia, não menciona o exame das evidências científicas correspondentes e apresenta informações limitadas acerca do esquema terapêutico a ser utilizado.

A seleção das tecnologias a serem utilizadas em nosso sistema de saúde, dados os interesses econômicos e o impacto do seu custo no orçamento do setor, deve implicar a avaliação cuidadosa dos efeitos dessas tecnologias como subsídio para decidir sobre a conveniência de sua implantação e em que condições, do ponto de vista da população. Por esse motivo, torna-se importante como ponto de partida para o exame da evidência, a análise ou a elaboração de revisões sistemáticas sobre a eficácia e segurança de cada uma dessas tecnologias.

A revisão sistemática é uma revisão de estudos, através de uma abordagem sistemática, com o objetivo de reduzir viés. Elas contribuem para integrar informações de diversos estudos, de forma adequada, fornecendo uma base científica para a tomada de decisão racional no setor saúde. A meta-análise é uma forma de revisão sistemática na qual ocorre uma análise estatística que combina os resultados de estudos independentes com o objetivo de se extrair uma medida de efeito sumária, possibilitando a melhoria da estimativa do tamanho do efeito para os desfechos primários e para subgrupos, o que pode ser proveitoso para a tomada de decisão sobre o uso de uma tecnologia 7.

O objetivo do presente trabalho foi o de analisar a evidência científica disponível sobre os efeitos da antibioticoprofilaxia no caso das cesarianas e, se necessário, elaborar revisão sistemática e eventual meta-análise, no sentido de fornecer subsídios atualizados para a tomada de decisão e o eventual aperfeiçoamento de diretrizes clínicas sobre $\mathrm{o}$ assunto.

\section{Metodologia}

\section{Evidências básicas}

Estudos experimentais apresentaram as bases científicas iniciais para o uso da antibioticopro- filaxia. Os resultados do ensaio de Burke 8, com porquinhos-da-Índia, indicaram que o antimicrobiano deve estar circulando e presente nos tecidos no momento em que ocorrer a incisão e a contaminação bacteriana do sítio operatório. Em 1980, Shapiro et al. ${ }^{9}$ confirmaram os achados de Burke, encontrando diferença significativa entre o diâmetro da lesão dos animais que receberam o antimicrobiano 1 hora antes da inoculação intradérmica de bactérias e o daqueles que receberam a droga 4 horas após a inoculação ou não receberam a droga. Dois estudos bem desenhados, em humanos, apóiam os achados relativos à importância da cobertura antimicrobiana no momento da cirurgia 10,11.

\section{Análise dos fatores prognósticos \\ de infecção pós-cesariana}

A análise dos estudos sobre fatores prognósticos de infecção cesariana é a etapa que precedeu a revisão dos ensaios sobre os efeitos da antibioticoprofilaxia, no sentido de permitir um exame mais apropriado da qualidade dos ensaios clínicos e da heterogeneidade clínica entre os ensaios.

Os estudos bem desenhados, prospectivos, revisados, que utilizaram análise multivariada permitiram identificar a ocorrência e a duração da ruptura de membrana, a idade materna menor que 20-25 anos, o trabalho de parto e ainda a vaginose bacteriana como fatores prognósticos importantes de endometrite 12,13,14. A variável trabalho de parto pode expressar ou mediar, junto com ruptura prematura de membrana, a presença de microorganismos relevantes na vagina e a contaminação ascendente (vaginose bacteriana). Os estudos não esclareceram a correlação entre idade jovem e infecção pós-cesariana. Os fatores prognósticos identificados foram focalizados no exame das evidências.

\section{Revisão da evidência científica sobre antibioticoprofilaxia em cesarianas}

Para a identificação das revisões sistemáticas de antibioticoprofilaxia em cesarianas foram realizadas buscas em bases de dados eletrônicas (The Cochrane Controlled Trials Register e MEDLINE) e em referências bibliográficas, resultando na seleção preliminar de três revisões sistemáticas: Smaill \& Hofmeyr 15, Chelmow et al. 16 e Hopkins \& Smaill 17. As duas primeiras analisaram ensaios que comparavam antimicrobiano com placebo ou não tratamento e a última, ensaios comparativos de esquemas de antibioticoprofilaxia. 


\section{- Avaliação das meta-análises disponíveis}

As meta-análises disponíveis foram avaliadas segundo a qualidade e a pertinência, tendo sido utilizada para esse fim uma lista de critérios derivada dos estudos de Sacks et al. 18 e Oxman 19, à qual foram acrescentados tópicos específicos.

Nenhum dos estudos investigou a possibilidade de viés de publicação. As meta-análises de Smaill \& Hofmeyr 15 e Hopkins \& Smaill 17 incluíram ensaios randomizados mas sem exigência quanto ao uso de placebo ou de cegamento do avaliador. A meta-análise de Smaill \& Hofmeyr 15 incluiu ensaios com intervenções de validade questionável ou especificação insatisfatória, como, por exemplo, estudos com intervenções com tempo superior a 24 horas e irrigação da cavidade uterina.

A avaliação da qualidade dos ensaios foi insatisfatória nas meta-análises de Smaill \& Hofmeyr 15 e de Hopkins \& Smaill 17, que utilizaram o "Standard Cochrane Criteria", o qual considera apenas critérios relativos ao cegamento da alocação. Ainda assim, aparentemente esses critérios não subsidiaram a exclusão de ensaios. Além disso, não foram mencionadas análises de sensibilidade.

A investigação da heterogeneidade clínica enquanto possível fonte de heterogeneidade de resultados, para efeito de combinabilidade de ensaios ${ }^{20}$, não foi efetuada em nenhuma das três meta-análises. As revisões também não informam se foram sistematicamente investigadas a questão das perdas e a forma de análise dos resultados (intenção de tratar/tratamento efetivo).

Em resumo, as três meta-análises selecionadas apresentaram limitações metodológicas em relação às características dos ensaios por elas incluídos e à sua execução, não sendo suficientemente válidas, enquanto evidência, para subsidiar a tomada de decisão em relação ao problema em pauta.

\section{- Elaboração das presentes revisões sistemáticas/meta-análises}

Para realizar as presentes revisões sistemáticas foram elaborados protocolos que são sumarizados a seguir. A estratégia de busca para ambas as revisões sistemáticas foi elaborada com base no Cochrane Reviewers' Handbook versão 4.1 (http://www.cochrane.org/resources/handbook/). As buscas foram efetuadas no mês de fevereiro de 2005. A pesquisa no MEDLINE foi adaptada pelo uso de palavras truncadas relacionadas à condição (cesar, caesar) e às intervenções (antibiot, antimicr, prophyla), que também foram utilizadas na pesquisa na base de dados da
Cochrane Library. Além disso, foram realizadas pesquisas em referências bibliográficas de artigos de interesse (revisões, ensaios clínicos e livros) e em anais de congressos na área de obstetrícia. A partir da referida estratégia de busca, foram encontrados 468 ensaios clínicos. Os ensaios foram selecionados com base nos seguintes critérios:

a) comparar regimes de antibioticoprofilaxia versus placebo ou comparar esquemas de antibioticoprofilaxia - dose única versus doses múltiplas do mesmo antibiótico e classes/gerações de antibióticos - em mulheres em geral (sem patologia específica) submetidas à cesariana;

b) Ser um ensaio clínico controlado randomizado e duplo-cego;

c) Ter iniciado a profilaxia pela via parenteral em até duas horas antes do início da cirurgia ou no momento do clampeamento do cordão, durante a cirurgia, tendo sido consideradas intervenções válidas os esquemas profiláticos com duração de até 24 horas;

d) Analisar ambos os desfechos: infecção de ferida cirúrgica (IFC) e endometrite, subtipos de infecção pós-cesariana;

e) Ser um antibiótico com cobertura antimicrobial relativamente adequada para ambos sub-tipos de infecção pós-cesariana e sem problemas relevantes de segurança.

Dos 468 ensaios pré-selecionados, 389 foram excluídos através da análise dos títulos e dos resumos porque focalizavam pacientes com comorbidades específicas ou não se referiam à antibioticoprofilaxia ou porque seu desenho não era válido. Restaram então 79 ensaios, dos quais 56 se referiam a estudos que comparavam antibioticoprofilaxia e placebo, 14 comparavam esquemas de dose única e doses múltiplas e 15 ensaios comparavam esquemas de antimicrobianos diferentes. A partir de um exame preliminar da metodologia dos 56 ensaios placebocontrolados, foram excluídos 29 ensaios, por não analisarem os desfechos endometrite e IFC, ou por não informarem se foram cegados/randomizados, ou porque o esquema profilático tinha duração superior a 24 horas, restando 27 ensaios placebo-controlados.

Dos 14 ensaios comparativos de doses, foram excluídos: seis porque comparavam doses de antimicrobianos diferentes, dois porque analisavam apenas endometrite e três porque nada informavam sobre cegamento ou randomização, restando três ensaios.

Dos 15 ensaios comparativos de tipos de antimicrobianos, foram excluídos: um por não analisar ambos os desfechos endometrite e IFC, um por não informar sobre cegamento/randomização, e um por duração do esquema profilático acima de 24 horas, restando 12 ensaios. 
Desses, sete apresentavam comparação entre cefalosporinas de 1a e 2a geração, dois comparavam cefalosporinas de 1a e $3 \underline{a}$ geração, e os quatro outros apresentavam comparações entre antimicrobianos diversos (penicilina, cefalosporina, gentamicina e clavulanato), não sendo interessante agrupá-los. Decidimos então analisar os ensaios comparativos entre cefalosporinas de la e 2 a geração.

Procedeu-se ainda a uma avaliação mais apurada da pertinência e a um exame mais detalhado da qualidade dos ensaios, com o objetivo de avaliar a força da evidência produzida por cada estudo e a validade de sua inclusão na meta-análise. Para esse fim, utilizou-se uma lista de critérios elaborada a partir dos trabalhos de Altman 7 e de Oxman ${ }^{19}$, considerando a qualidade do desenho, a validade das intervenções, a delimitação dos desfechos e a análise de dados. Ambas as fases de seleção e de análise sistemática da qualidade dos ensaios foram realizadas antes da leitura da seção de resultados dos ensaios, procurando-se evitar a consideração desses resultados naqueles procedimentos. Essas etapas foram feitas de forma independente pelos autores, havendo decisão por consenso nos casos contraditórios.

As definições de IFC e de endometrite foram avaliadas tendo como referência, respectivamente, as propostas do Centers for Disease Control and Prevention (CDC) de 1992 21, para infecção incisional superficial e profunda, e de 1999 22, para endometrite.

Avaliamos ainda a heterogeneidade clínica entre os estudos antes de analisarmos os resultados dos ensaios, com o objetivo de avaliar se a medida sumária do efeito deveria ser estimada. A heterogeneidade clínica refere-se à diferença entre os estudos quanto às características das participantes (critérios de inclusão e exclusão, prognóstico), das intervenções (tipo, dose, duração) e dos desfechos analisados (definição, tempo de seguimento) 20 .

Os protocolos previram ainda a análise de subgrupo (intra-ensaio, de preferência) considerando as variáveis prognósticas ocorrência de ruptura de membrana e de trabalho de parto, tendo sido definidas como cesáreas eletivas, as cirurgias em que as mulheres não desencadearam trabalho de parto e possuíam membranas intactas e, como cesáreas não eletivas, as cirurgias feitas em mulheres que estavam em trabalho de parto e/ou apresentavam ruptura de membrana. Foi prevista ainda análise de sensibilidade para avaliar o impacto, no efeito sumário, de limitações na qualidade do desenho/consecução/relato dos ensaios consideradas importantes para esse tipo de ensaio.

\section{Análise estatística}

A análise dos dados dos ensaios foi feita utilizando-se o programa estatístico RevMan Analyses versão 4.2 (http:/ /www.cc-ims.net/RevMan). Foram calculados as razões de taxas (RT) e os intervalos de confiança com nível de significância de 95\% (IC95\%), por meio dos modelos de efeitos fixos (MEF) e de efeitos randômicos (MER). O cálculo da heterogeneidade estatística foi realizado através do teste do $\chi^{2}$, sendo $\mathrm{p}>0,1$ o nível de significância utilizado para considerar homogêneos os resultados.

\section{Resultados}

\section{Revisão sistemática e principais problemas encontrados na execução da meta-análise dos ensaios placebo-controlados}

\section{a) Possível viés de publicação}

A análise do gráfico de funil, tanto para o desfecho endometrite quanto para IFC, revelou a existência de assimetria de estudos, com quase ausência de estudos negativos na base do funil, ou seja, de estudos de tamanho reduzido.

\section{b) Falhas do desenho, execução e análise dos ensaios}

Os 27 ensaios, em geral, apresentaram claramente os critérios de inclusão/exclusão das participantes. Embora tenham referido a realização da randomização, não informaram sobre a geração da seqüência de alocação. O cegamento da alocação não foi explicitado na maioria dos ensaios, embora a maioria deles haja informado que o placebo tinha características semelhantes à droga. A maioria dos ensaios apresentou uma tabela pós-randomização; no entanto, a estrutura das variáveis apresentadas não incluiu, em geral, as variáveis prognósticas identificadas pelos estudos de fatores prognósticos, referidos anteriormente. Apesar da limitação das informações, identificamos desequilíbrio nos oito ensaios que reportaram sobre as variáveis ocorrência de ruptura e de trabalho de parto e duração média da ruptura; no entanto, para o conjunto dos ensaios, não identificamos nenhum grupo (placebo ou tratado) preferencialmente favorecido. Todos os ensaios referiram a realização de cegamento do tipo duplo-cego, embora 15 dos 27 ensaios não tenham explicitado o cegamento do avaliador. Em nove ensaios, a profilaxia foi iniciada até uma hora antes do início da cirurgia. Nos 19 ensaios restantes, a intervenção foi iniciada logo após o 
clampeamento do cordão. Apenas cinco ensaios forneceram informações relevantes sobre cointervenções.

Foram observadas variações na especificidade e sensibilidade das definições dos desfechos. Todas as definições, todavia, foram consideradas válidas, com base no protocolo, com exceção da definição do estudo de Young et al. 23, que foi excluído, por considerar endometrite como febre e ausência de evidência clínica ou laboratorial de infecção em outro sítio. Onze dos 27 ensaios não informaram sobre exclusões ou perdas pós-randomização e destes, nove não informaram sobre a forma de análise de dados. Dois ensaios tiveram perdas superiores a $20 \%$, boa parte relacionada a erros na administração da profilaxia, e só permitem a análise por tratamento efetivo 24,25 . Apenas seis estudos apresentaram os dados por intenção de tratar ou forneceram informação que possibilitou a análise por intenção de tratar, 16 apresentaram os dados por tratamento efetivo ou apresentaram dados que possibilitaram essa análise, sendo que destes apenas quatro permitiram também a análise por intenção de tratar.

\section{c) Heterogeneidade clínica entre os ensaios}

Observou-se a inclusão de pacientes com estruturas diferenciadas quanto à presença dos fatores prognósticos trabalho de parto e ruptura de membrana. Além disso, os dados disponíveis indicaram heterogeneidade entre os estudos quanto ao tempo de ruptura de membrana. Houve ainda variação nas intervenções testadas em relação aos espectros dos antimicrobianos, início da intervenção e número de doses dos antimicrobianos, caracterizando a presença de heterogeneidade clínica.
Outra fonte de heterogeneidade relacionada à intervenção referiu-se ao tempo de duração da profilaxia. Dentre os ensaios analisados, apenas nove utilizaram esquemas profiláticos de dose única, o restante utilizou regimes de múltiplas doses, com esquemas de duração variados (4-12 horas).

Em relação à medida do desfecho, houve variação entre os ensaios nas medidas de endometrite e de infecção da ferida. Houve também variação entre os ensaios quanto a abranger ou não o subtipo infecção incisional superficial.

\section{- Estimativa da eficácia da antibioticoprofilaxia (ensaios placebo-controlados)}

Os 27 ensaios totalizaram 4.470 pacientes. Foi observada heterogeneidade estatística significativa para os ensaios por intenção de tratar para o desfecho IFC. Em todos os conjuntos (global, intenção de tratar, tratamento efetivo e indefinido) foram observadas RT favoráveis à antibioticoprofilaxia, com eficácia muito semelhante para endometrite (65\%, MEF/MER) e IFC (65\%/MER), estatisticamente significativas. As diferenças de taxas (DT) dos ensaios por intenção de tratar foram estatisticamente significativas, $3 \%$ e $5.6 \%$, e para o conjunto dos ensaios, $11 \%$ e $5 \%$, respectivamente, para endometrite e para IFC (Tabela 1 e Figura 1).

\section{- Análise de subgrupos}

A análise de subgrupo segundo a presença/ausência dos fatores trabalho de parto e ruptura de membrana (cesáreas eletivas e não eletivas) intra-ensaios não foi possível, já que os ensaios que incluíram ambos os subtipos de pacientes

Tabela 1

Efeitos sumários da antibioticoprofilaxia para os desfechos endometrite e infecção da ferida cirúrgica (IFC)

(ensaios placebo-controlados), quanto à forma de análise dos dados.

\begin{tabular}{|c|c|c|c|c|c|c|}
\hline \multirow[b]{2}{*}{ Forma de análise dos dados } & \multirow[b]{2}{*}{ Ensaio (N) } & \multicolumn{5}{|c|}{ RT (sumário) } \\
\hline & & $\chi^{2}$ & Desfecho & MEF & MER & DT (sumário) \\
\hline \multirow[t]{2}{*}{ Intenção de tratar } & 6 & $p=0,59$ & Endometrite & $0,34(0,22-0,54)$ & $0,35(0,22-0,56)$ & $3,0(1,4-4,6)$ \\
\hline & 6 & $p=0,004$ & IFC & $0,36(0,24-0,54)$ & $0,38(0,20-0,71)$ & $5,6(3,3-7,8)$ \\
\hline \multirow[t]{2}{*}{ Efetivamente tratados } & 16 & $p=0,63$ & Endometrite & $0,35(0,27-0,44)$ & $0,36(0,27-0,45)$ & $10,0(8,0-12,0)$ \\
\hline & 16 & $p=0,46$ & IFC & $0,31(0,22-0,45)$ & $0,32(0,22-0,49)$ & $6,0(4,0-8,0)$ \\
\hline \multirow[t]{2}{*}{ Não informado } & 9 & $p=0,45$ & Endometrite & $0,30(0,20-0,44)$ & $0,31(0,29-0,60)$ & $2,2(0,3-4,1)$ \\
\hline & 9 & $p=0,61$ & IFC & $0,33(0,12-0,73)$ & $0,34(0,10-0,78)$ & $1,1(-0,4-2,5)$ \\
\hline \multirow[t]{2}{*}{ Todos os ensaios } & 27 & $p=0,53$ & Endometrite & $0,33(0,27-0,40)$ & $0,36(0,29-0,43)$ & $11,0(9,0-12,0)$ \\
\hline & 27 & $p=0,85$ & IFC & $0,32(0,24-0,43)$ & $0,35(0,25-0,47)$ & $5,0(4,0-6,0)$ \\
\hline
\end{tabular}


Figura 1

Taxas de endometrite em ensaios placebo-controlados de antibioticoprofilaxia em cesarianas, modelo de efeitos fixos (Mantel \& Haenszel).

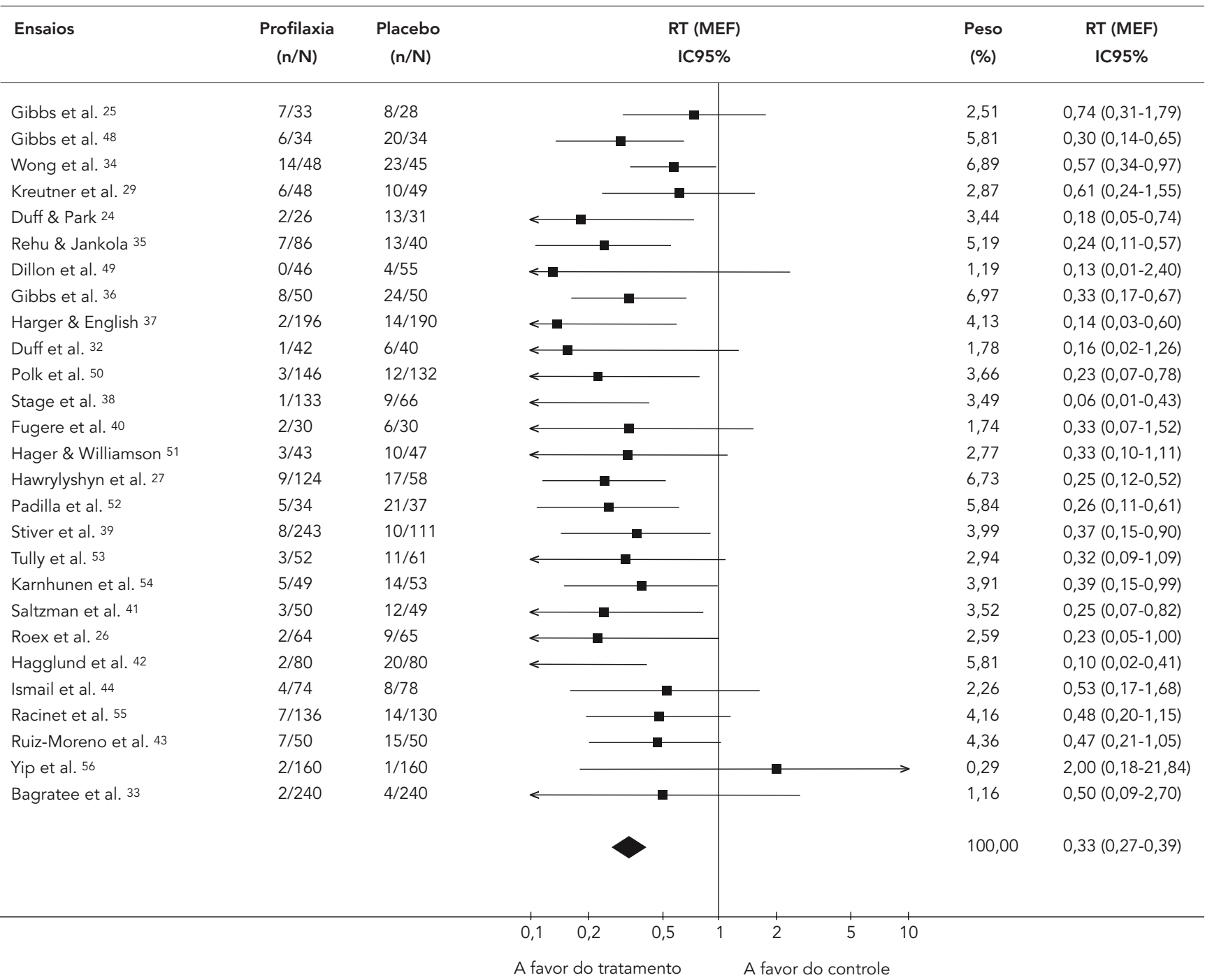

Teste de heterogeneidade: $\chi^{2}=24.75, d f=26(p=0,53), 12=0 \%$

Teste de efeito sumário: $Z=11.42(p=0,00001)$

não apresentaram os resultados por subtipo. A análise de subgrupo foi então efetuada entre ensaios, utilizando as informações disponíveis nos ensaios.

Dentre os 27 ensaios, apenas dois apresentaram resultados para cesáreas eletivas, 12 para não eletivas e 13 não permitiram a análise de seus resultados segundo cesáreas eletivas ou não. Para o desfecho endometrite, a combinação dos resultados dos dois ensaios de cesáreas eletivas apresentou uma RT bastante favorável à antibioticoprofilaxia $(0,32)$, mas com intervalo amplo, próximo a um, sendo pouco relevante a DT encontrada $(1,4 \%)$ (Tabela 2$)$. Com relação à IFC, a razão e a diferença de taxas encontradas foram pouco relevantes e sem significância estatística.

Para o conjunto de 12 ensaios de cesáreas não eletivas, não foi encontrada heterogeneidade estatisticamente significativa, nem para endometrite nem para IFC (Tabela 2). A combinação dos resultados desses ensaios apontou a existência de benefício importante da antibioticoprofilaxia para a prevenção da endometrite $(\mathrm{RT}=0,32$; $\mathrm{DT}=$ $14 \%$ ) e da IFC (RT = 0,29; DT = 5\%) (Tabela 2). 
Efeitos sumários da antibioticoprofilaxia para os desfechos endometrite e infecção da ferida cirúrgica (IFC), segundo os subgrupos cesarianas eletivas e não eletivas (análise entre ensaios).

\begin{tabular}{|c|c|c|c|c|c|c|c|c|c|}
\hline \multirow[b]{2}{*}{ Referência } & \multirow[b]{2}{*}{$\begin{array}{c}\text { Ano de } \\
\text { publicação }\end{array}$} & \multicolumn{3}{|c|}{ Grupo tratado } & \multicolumn{3}{|c|}{ Grupo placebo } & \multicolumn{2}{|c|}{ RT (IC95\%) } \\
\hline & & $\mathbf{N}$ & Endometrite & IFC & $\mathbf{N}$ & Endometrite & IFC & Endometrite & IFC \\
\hline \multicolumn{10}{|l|}{ Cesarianas eletivas } \\
\hline Duff \& Park 24 & 1980 & 13 & 0 & - & 12 & 5 & - & $0,04(0,001-2,26)$ & - \\
\hline Duff et al. 32 & 1982 & 42 & 1 & 0 & 40 & 6 & 0 & $0,16(0,02-1,26)$ & $0,96(0,00-9,82)$ \\
\hline Bagratee et al. 33 & 2001 & 240 & 2 & 13 & 240 & 4 & 15 & $0,50(0,09-2,72)$ & $0,87(0,43-1,78)$ \\
\hline Total & & 282 & 3 & 13 & 280 & 10 & 15 & & \\
\hline \multicolumn{10}{|c|}{ Teste de heterogeneidade: } \\
\hline qui-quadrado & & & & & & & & $\chi^{2}=0,71 ; p=0,40$ & $\chi^{2}=0,68 ; p=0,42$ \\
\hline \multicolumn{10}{|c|}{ Razão de taxas: modelo } \\
\hline de efeitos fixos & & & & & & & & $0,29(0,09-0,92)$ & $0,87(0,43-1,77)$ \\
\hline \multicolumn{10}{|c|}{ Razão de taxas: modelo de } \\
\hline efeitos aleatórios & & & & & & & & $0,32(0,09-1,18)$ & $0,95(0,59-1,50)$ \\
\hline \multicolumn{10}{|c|}{ Diferença de taxas: modelo } \\
\hline de efeitos fixos & & & & & & & & $1,4(-0,7-3,4)$ & $0,3(-2,3-3,0)$ \\
\hline \multicolumn{10}{|l|}{ Cesarianas não eletivas } \\
\hline Wong et al. 34 & 1978 & 48 & 14 & 2 & 45 & 23 & 3 & $0,57(0,34-0,97)$ & $0,65(0,13-3,39)$ \\
\hline Duff \& Park 24 & 1980 & 13 & 2 & - & 19 & 8 & - & $0,37(0,09-1,45)$ & - \\
\hline Rehu \& Jahkola 35 & 1980 & 88 & 7 & 4 & 40 & 13 & 4 & $0,20(0,06-0,65)$ & $0,46(0,10-2,10)$ \\
\hline Gibbs et al. 36 & 1981 & 50 & 8 & 0 & 50 & 24 & 2 & $0,33(0,17-0,67)$ & $0,11(0,002-6,80)$ \\
\hline Harger \& English 37 & 1981 & 196 & 20 & 2 & 190 & 38 & 14 & $0,51(0,31-0,84)$ & $0,15(0,04-0,62)$ \\
\hline Stage et al. 38 & 1982 & 133 & 1 & 3 & 66 & 9 & 12 & $0,06(0,07-0,43)$ & $0,13(0,04-0,43)$ \\
\hline Stiver et al. 39 & 1983 & 243 & 8 & 6 & 111 & 10 & 17 & $0,47(0,17-1,34)$ & $0,12(0,03-0,48)$ \\
\hline Fugere et al. 40 & 1983 & 30 & 2 & 2 & 30 & 6 & 6 & $0,017(0,02-1,30)$ & $0,36(0,09-1,52)$ \\
\hline Hawrylyshyn et al. 27 & 1983 & 124 & 9 & 2 & 58 & 17 & 2 & $0,32(0,14-0,76)$ & $0,5(0,06-4,36)$ \\
\hline Saltzman et al. 41 & 1985 & 50 & 3 & 1 & 49 & 12 & 2 & $0,25(0,07-0,82)$ & $0,55(0,063-4,68)$ \\
\hline Hagglund et al. 42 & 1989 & 80 & 2 & 0 & 80 & 20 & 3 & $0,10(0,02-0,41)$ & $0,08(0,001-4,45)$ \\
\hline Ruiz-Moreno et al. 43 & & 50 & 7 & 1 & 50 & 15 & 4 & $0,47(0,21-1,05)$ & $0,06(0,07-0,03)$ \\
\hline Ismail et al. 44 & 1990 & 74 & 4 & 2 & 78 & 8 & 6 & $0,53(0,17-1,68)$ & $0,38(0,09-1,69)$ \\
\hline Total & & 1.179 & 87 & 25 & 866 & 203 & 75 & & \\
\hline \multicolumn{10}{|c|}{ Teste de heterogeneidade: } \\
\hline qui-quadrado & & & & & & & & $\chi^{2}=17,1 ; p=0,32$ & $\chi^{2}=5,74 ; p=0,84$ \\
\hline \multicolumn{10}{|c|}{ Razão de taxas: modelo de } \\
\hline efeitos fixos & & & & & & & & $0,32(0,24-0,41)$ & $0,29(0,18-0,47)$ \\
\hline \multicolumn{10}{|c|}{ Razão de taxas: modelo de } \\
\hline efeitos aleatórios & & & & & & & & $0,35(0,27-0,45)$ & $0,29(0,18-0,50)$ \\
\hline \multicolumn{10}{|c|}{ Diferença de taxas: modelo } \\
\hline de efeitos fixos & & & & & & & & $14,0(11,0-17,0)$ & $5,0(3,0-7,0)$ \\
\hline
\end{tabular}

\section{- Análises de sensibilidade}

Conforme previsto no protocolo, análises de sensibilidade foram realizadas em relação aos seguintes parâmetros: (a) momento de início da profilaxia; (b) equilíbrio entre os grupos em relação às variáveis prognósticas; e (c) perdas ou exclusões pós-randomização (se se relata perdas adequadamente e se tais perdas afetam a valida- de do estudo). Tanto as RT sumárias dos ensaios com início da antibioticoprofilaxia anterior à cirurgia, como as dos ensaios com equilíbrio para as variáveis prognósticas e as dos ensaios com execução considerada adequada (relato e validade), não foram significativamente diferentes das razões encontradas para o conjunto dos ensaios ou para o conjunto dos ensaios por intenção de tratar. 
Revisão sistemática e principais problemas encontrados na execução da meta-análise dos ensaios comparativos de dose única versus dose múltipla

a) Falhas do desenho, execução e análise dos ensaios

Embora tenham referido a realização de randomização, nenhum dos três ensaios explicitou o método utilizado. Os dados apresentados nas tabelas pós-randomização dos três estudos indicam a presença de desequilíbrio em relação à variável duração de ruptura de membrana em todos os estudos, favorecendo o grupo de múltiplas doses. Esse grupo de tratamento também foi favorecido em relação ao percentual de mulheres com anemia grave no estudo de Roex et al. 26. Em apenas um ensaio havia informação sobre cegamento da alocação. Os três ensaios relataram perdas ou exclusões pós-randomização, sendo que no ensaio de Hawrylyshyn et al. 27, com 124 pacientes randomizadas, houve seis exclusões por ocorrência de febre até oito horas após a cirurgia, requerendo antibioticoterapia, não sendo informado em que grupos ocorreram as exclusões. No estudo de Gall et al. 28 , houve 13 perdas em 116 , por erros na administração dos antimicrobianos, sendo explicitado em que grupos ocorreram. Os resultados foram apresentados apenas segundo tratamento efetivo, sendo possível produzir a análise por intenção de tratar apenas para o estudo de Gall et al. ${ }^{28}$.

\section{b) Heterogeneidade clínica entre os ensaios}

Os três ensaios comparativos de doses compreenderam populações com elevado percentual de pacientes de alto risco para infecção pós-cesariana. Não identificamos heterogeneidade clínica importante em relação às variáveis prognósticas, da forma em que foram relatadas. Os ensaios diferiram quanto à dose dos antimicrobianos usados, à duração dos esquemas de múltiplas doses e ao espectro dos antimicrobianos usados, indicando a presença de heterogeneidade clínica que poderia propiciar variação nos resultados entre os ensaios. Foram identificadas variações nas definições de IFC utilizadas pelos ensaios, embora as definições estivessem de acordo com os parâmetros considerados. O subtipo de infecção incisional superficial não foi avaliada em um dos estudos.

\section{- Estimativa da eficácia dos ensaios comparativos de número de doses}

Os três ensaios comparativos analisados contaram com um total de pacientes tratadas próxi- mo a 400. Não foi observada heterogeneidade estatisticamente significativa entre os ensaios, nem para o desfecho endometrite nem para IFC (Tabela 3). A razão de taxas sumária, segundo tratamento efetivo (única análise disponível), foi favorável ao grupo de múltiplas doses, para o desfecho endometrite, todavia, com intervalo de confiança amplo, próximo a um, sendo a diferença de taxas de $6,1 \%$, também com amplo intervalo de confiança (Tabela 3). Em relação à IFC, a RT sumária também foi favorável ao grupo de múltiplas doses, embora sem significância estatística, sendo a DT, irrelevante.

Revisão sistemática e principais problemas encontrados na execução da meta-análise dos ensaios comparativos de antimicrobianos (cefalosporinas de 1ạ geração versus cefalosporinas de 2a geração)

\section{a) Falhas do desenho, execução e análise dos ensaios}

Os sete ensaios referiram ser randomizados mas não informaram sobre os procedimentos empregados. A tabela pós-randomização não foi apresentada em dois ensaios 29,30; naqueles que a apresentaram, não identificamos desequilíbrio em relação às variáveis ocorrência/duração média de trabalho de parto e ruptura de membrana, não havendo informação sobre vaginose bacteriana ou idade materna. Quatro ensaios não informaram sobre perdas, exclusões pós-randomização e/ou crossover nem sobre a forma de análise dos dados. Apenas um dos sete ensaios apresentou os dados por intenção de tratar; dois analisaram o tratamento efetivo, não permitindo reconstituir a análise para intenção de tratar; quatro ensaios não mencionaram perdas/exclusões, embora alguns ensaios apresentassem grupos de tratamento de tamanhos bastante diferentes, nem informaram sobre a forma de análise dos dados.

\section{b) Heterogeneidade clínica entre os ensaios}

Os ensaios compreenderam populações com elevado percentual de pacientes de alto risco para infecção. Dos sete ensaios analisados, quatro utilizaram esquemas profiláticos de dose única e o restante utilizou regimes de múltiplas doses, com esquemas de duração de cerca de 12 horas. Houve variação nas definições de endometrite e IFC utilizadas pelos ensaios, embora todas estivessem de acordo com os parâmetros definidos. Além disso, o subtipo infecção incisional superficial não foi considerado em dois dos estudos. 
Efeitos sumários de ensaios clínicos comparativos de dose única e doses múltiplas para os desfechos endometrite e infecção da ferida cirúrgica (IFC).

\begin{tabular}{|c|c|c|c|c|c|c|c|c|c|}
\hline \multirow[b]{2}{*}{ Referência } & \multirow[b]{2}{*}{$\begin{array}{c}\text { Ano de } \\
\text { publicação }\end{array}$} & \multicolumn{3}{|c|}{ Doses múltiplas } & \multicolumn{3}{|c|}{ Dose única } & \multicolumn{2}{|c|}{ RT (IC95\%) } \\
\hline & & $\mathbf{N}$ & Endometrite & IFC & $\mathbf{N}$ & Endometrite & IFC & Endometrite & IFC \\
\hline Hawrylyshyn et al. 27 & 1983 & 60 & 3 & 1 & 64 & 6 & 1 & $0,53(0,14-2,04)$ & $1,07(0,09-2,42)$ \\
\hline Roex et al. 26 & 1986 & 72 & 1 & 1 & 66 & 5 & 7 & $0,18(0,22-1,53)$ & $0,16(0,20-1,03)$ \\
\hline Gall \& Hill 28 & 1987 & 56 & 3 & 1 & 60 & 8 & 0 & $0,40(0,11-1,44)$ & $5,36(0,07-86,64)$ \\
\hline Total & & 188 & 7 & 3 & 190 & 19 & 8 & & \\
\hline \multicolumn{10}{|c|}{ Teste de heterogeneidade: } \\
\hline qui-quadrado & & & & & & & & $\chi^{2}=0,70 ; p=0,71$ & $\chi^{2}=2,96 ; p=0,23$ \\
\hline \multicolumn{10}{|l|}{ Medida sumária: modelo } \\
\hline de efeitos fixos & & & & & & & & RT: 0,40 (0,17-0,93) & RT: 0,98 (0,43-2,21) \\
\hline \multicolumn{10}{|l|}{ Medida sumária: modelo } \\
\hline de efeitos aleatórios & & & & & & & & RT: $0,48(0,17-0,98)$ & RT: $1,08(0,38-2,32)$ \\
\hline \multicolumn{10}{|l|}{ Diferença de taxas: } \\
\hline modelo de efeitos fixos & & & & & & & & DT: $6,1(1,2-10,9)$ & DT: $3,0(-1,0-7,0)$ \\
\hline
\end{tabular}

\section{- Estimativa da eficácia em ensaios comparativos de diferentes antimicrobianos}

Para os sete ensaios analisados, que somaram 1.302 pacientes, não foi observada heterogeneidade estatística significativa nas análises para endometrite ou IFC. As RT sumárias não mostraram evidência de benefício superior da cefalosporina de 2a geração em relação à cefalosporina de 1a geração ( 4 tratamento efetivo, 4 indefinido) (Tabela 4).

\section{Discussão}

As três meta-análises que já estavam disponíveis sobre antibioticoprofilaxia em cesarianas analisadas apresentaram limitações importantes para serem utilizadas como subsídio para decisões e diretrizes sobre o assunto. As revisões sistemáticas objeto do presente trabalho focalizaram a análise detalhada da qualidade e pertinência dos ensaios e das fontes de heterogeneidade dos resultados, buscando também esclarecer se, em que momento, por quanto tempo (número de doses) e quais os antimicrobianos deveriam ser usados para evitar infecção pós-cesariana.

A análise dos estudos sobre fatores prognósticos pretendeu apoiar a análise dos ensaios clínicos no que se refere a questões relacionadas a viés e confundimentos. $\mathrm{O}$ exame dos achados dos estudos preliminares em humanos e sobre a flora vaginal e da parede abdominal subsidiou a análise da evidência, dando suporte à análise da plausibilidade biológica dos esquemas antimicrobianos testados (início, duração e tipo de antimicrobiano).

Quanto à existência de viés de publicação, o gráfico de funil dos ensaios placebo-controlados revelou uma grande assimetria. Esse aspecto aponta a possibilidade de que estudos com resultados negativos, principalmente de pequeno porte, não tenham sido publicados, o que implicaria a superestimativa do efeito. Outras explicações para a assimetria poderiam ser falhas metodológicas e diferença de prognóstico dos pacientes dos pequenos ensaios.

Quanto ao desenho dos ensaios, as informações sobre os procedimentos de randomização e sobre o cegamento da alocação foram escassas. Já sobre o cegamento do avaliador, ponto crítico para o conjunto de ensaios analisados, visto que algumas medidas de desfecho englobaram critérios subjetivos, todos os ensaios incluídos informaram apenas ser duplo-cegos. Assumimos, por isso, que houve cegamento da avaliação, considerando inclusive a possibilidade de o responsável pela intervenção, o cirurgião, e o avaliador serem a mesma pessoa.

Os dados apresentados nas tabelas pós-randomização disponíveis foram, na maioria dos ensaios, insuficientes para subsidiar a análise da distribuição dos fatores prognósticos mais relevantes identificados, e omissos quanto à informação sobre a freqüência de vaginose bacteriana e o percentual de pacientes com menos de 20-25 anos, limitando bastante a análise do sucesso da randomização. 
Efeitos sumários de ensaios clínicos comparativos de profilaxia com cefalosporinas de 1a geração versus cefalosporinas de 2a geração para os desfechos endometrite e infecção da ferida cirúrgica (IFC).

\begin{tabular}{|c|c|c|c|c|c|c|c|c|c|}
\hline \multirow[b]{2}{*}{ Referência } & \multirow[b]{2}{*}{$\begin{array}{c}\text { Ano de } \\
\text { publicação }\end{array}$} & \multicolumn{3}{|c|}{ Grupo tratado } & \multicolumn{3}{|c|}{ Grupo placebo } & \multicolumn{2}{|c|}{ RT (IC95\%) } \\
\hline & & $\mathbf{N}$ & Endometrite & IFC & $\mathbf{N}$ & Endometrite & IFC & Endometrite & IFC \\
\hline Kreutner et al. 29 & 1979 & 48 & 15 & 1 & 43 & 10 & 1 & $0,74(0,37-1,48)$ & $1,12(0,07-17,31)$ \\
\hline Harger \& English 37 & 1981 & 63 & 4 & 2 & 63 & 9 & 0 & $2,25(0,73-6,93)$ & $0,20(0,01-4,08)$ \\
\hline Fugere et al. 40 & 1983 & 30 & 1 & 0 & 30 & 1 & 2 & $1,00(0,07-15,26)$ & $5,00(0,25-99,95)$ \\
\hline Stiver et al. 39 & 1983 & 119 & 3 & 4 & 124 & 4 & 2 & $1,28(0,29-5,60)$ & $0,48(0,09-1,59)$ \\
\hline Duff et al. 45 & 1987 & 196 & 19 & 0 & 103 & 13 & 0 & $1,30(0,67-2,53)$ & - \\
\hline Carlson \& Duff 46 & 1990 & 192 & 37 & 3 & 185 & 39 & 3 & $1,09(0,73-1,64)$ & $1,04(0,21-5,08)$ \\
\hline Peterson et al. 47 & 1990 & 47 & 6 & 0 & 59 & 6 & 2 & $0,80(0,27-2,31)$ & $4,00(0,20-81,36)$ \\
\hline Total & & 695 & 85 & 10 & 607 & 82 & 10 & & \\
\hline \multicolumn{10}{|c|}{ Teste de heterogeneidade: } \\
\hline qui-quadrado & & & & & & & & $\chi^{2}=3,45 ; p=0,75$ & $\chi^{2}=3,75 ; p=0,59$ \\
\hline \multicolumn{10}{|l|}{ Medida sumária: modelo } \\
\hline de efeitos fixos & & & & & & & & $\mathrm{RT}: 1,11(0,84-1,46)$ & RT: 0,98 (0,43-2,21) \\
\hline \multicolumn{10}{|l|}{ Medida sumária: modelo } \\
\hline de efeitos aleatórios & & & & & & & & RT: 1,09 (0,82-1,44) & RT: $0,94(0,38-2,32)$ \\
\hline \multicolumn{10}{|l|}{ Diferença de taxas: } \\
\hline modelo de efeitos fixos & & & & & & & & DT: $3,0(-1,0-7,0)$ & DT: $3,0(-1,0-7,0)$ \\
\hline
\end{tabular}

Com relação ao momento de início da profilaxia, embora em nove ensaios a profilaxia tenha sido iniciada até uma hora antes do início da cirurgia, possibilitando o alcance de níveis teciduais de antimicrobianos no decorrer da cirurgia, nos 18 ensaios restantes, a intervenção só foi iniciada após o clampeamento do cordão. Todavia, a análise de sensibilidade correspondente não indicou variação relevante dos efeitos sumários estudados.

Os antimicrobianos testados, em geral, apresentam boa cobertura para aeróbios gram-positivos, excetuando-se as cefalosporinas, com relação ao grupo dos enterococcos. Em relação à cobertura de aeróbios gram-negativos, os antimicrobianos estudados apresentam um pouco mais de variação em suas coberturas, particularmente em relação a Enterobacter sp. e Klebsiella sp. Alguns dos antimicrobianos usados não apresentam cobertura para bactérias anaeróbias, o que acontece também em relação a bactérias aeróbias, sendo que os desfechos estudados podem ser causados por ambos os tipos de bactérias.

Para a definição de endometrite e IFC foram utilizados critérios clinicamente mais e menos precoces (mais ou menos específicos) pelos diferentes ensaios, compatíveis, todavia, com a diversidade de delimitações proposta pelo CDC 21,22, em 1992 e 1999. O ensaio de Young et al. 23 foi excluído, pois a definição de endometrite foi considerada não válida, por ser muito sensível, já que os autores não caracterizaram febre e não apresentaram critérios adicionais para a classificação de endometrite.

Nove dos 27 ensaios placebo-controlados não informaram sobre exclusões pós-randomização, crossover ou perdas, e nem se a análise foi realizada por intenção de tratar ou por tratamento efetivo. A grande maioria das perdas relatadas foi devida a erros na administração da droga, havendo casos de histerectomia, alergia após a 1a dose e complicações não infecciosas durante o parto. A maioria dos ensaios que informou perdas/exclusões não especificou os grupos em que ocorreram, inviabilizando a análise por intenção de tratar. Os ensaios, em geral, não informaram sobre co-intervenções. Tais falhas limitaram a avaliação da execução e da forma de análise de dados dos ensaios.

Dada a citada omissão/pobreza de informações sobre variáveis prognósticas relevantes, o exame da heterogeneidade clínica entre os estudos para avaliar diferenças nas populações incluídas, com o objetivo de subsidiar a decisão sobre a validade de se estimar uma medida sumária dos resultados, foi limitado. Nos ensaios em que essas informações estavam disponíveis, observaram-se diferenças entre ensaios nas pa- 
cientes incluídas, particularmente para o tempo de exposição ao fator ruptura de membrana. Embora os resultados do teste de heterogeneidade estatística tenham sido negativos para os ensaios placebo-controlados, a heterogeneidade clínica acima referida é consistente com as observações de que tais testes têm baixa sensibilidade para detectar heterogeneidade clínica e devem ser interpretados com cautela.

Os ensaios apresentaram, em geral, critérios mais homogêneos, para o desfecho endometrite que para IFC. No entanto, seis ensaios não apresentaram os critérios para IFC e quatro não apresentaram os critérios utilizados para endometrite. Essa heterogeneidade, inclusive a omitida, pode ter sido uma fonte relevante de heterogeneidade de resultados, principalmente quanto à IFC. Também a heterogeneidade em relação aos esquemas terapêuticos utilizados pelos estudos, acima mencionada, pode explicar discrepâncias nos resultados dos estudos.

Apenas o estudo de Duff \& Park 24 apresentou resultados estratificados para os subgrupos relativos às variáveis prognósticas trabalho de parto e ruptura de membrana (cesárias não eletivas/ eletivas), o que inviabilizou a análise intra-ensaios, de maior validade. A análise entre ensaios para cesárias eletivas e não eletivas sugeriu maior eficácia e benefício absoluto (diferença de taxas) substancialmente mais elevado para cesárias não eletivas.

Embora as análises de sensibilidade efetuadas (para início tardio da profilaxia, desequilíbrio de prognóstico e para execução e análises inadequadas) não tenham mostrado diferenças de resultados em comparação tanto com os do conjunto de ensaios quanto com os resultados por intenção de tratar, apenas dois dentre os 27 ensaios, ou seja, menos de $10 \%$, apresentaram tanto equilíbrio para as variáveis prognósticas quanto execução considerada adequada e dados por intenção de tratar.

A comparação entre regimes de dose única e múltiplas doses é bastante relevante, pois a utilização de regimes de múltiplas doses implica aumento de custos. A análise da distribuição das variáveis prognósticas nos três ensaios, embora limitada, indicou um favorecimento do grupo de múltiplas doses em todos os estudos. Foram relatadas perdas/exclusões relevantes em dois ensaios e somente em um dos ensaios foi possível produzir a análise dos resultados segundo intenção de tratar. Ainda assim, as de taxas sumárias não mostraram benefício para o grupo de múltiplas doses.

Quanto aos ensaios comparativos de antimicrobianos, houve limitações nas informações sobre prognóstico, o que dificultou a análise do equilíbrio entre os grupos e limitou a análise da heterogeneidade, entre estudos. Também houve falhas importantes no relato sobre perdas e sobre forma de análise, limitando o exame da qualidade desses estudos e invalidando análises de sensibilidade.

É interessante notar que o uso de antibioticoterapia durante o pré-natal para combater a vaginose bacteriana e indiretamente, a endometrite pós-cesariana tem apresentado eficácia limitada 3 , possivelmente, em parte, pela natureza multi-organismo da invasão, mesmo com o esquema mais recente que associa metronidazol e eritromicina. O uso de probióticos, lactobacilos específicos, para combater a vaginose bacteriana também vem sendo estudado em mulheres não grávidas, não havendo ainda relatos quanto ao desfecho endometrite póscesariana 31 .

Tendo em vista o aleitamento materno, calculou-se a RT dos 23 ensaios placebo-controlados de antimicrobianos com meia vida curta (menor que duas horas), que não são excretados pelo leite ou têm baixa excreção, excluindo-se, portanto, os ensaios de kanamicina, metronidazol e tinidazol, que foi de $0,34(0,25-0,47)$ para IFC e de 0,31 $(0,25-0,38 / \mathrm{MEF})$ para endometrite, semelhantes aos resultados gerais.

\section{Conclusões}

O resultado da combinação dos ensaios placebocontrolados indica a existência de benefício importante da antibioticoprofilaxia na prevenção da endometrite e IFC em cesarianas, com eficácia da ordem de $65 \%$ para ambos os desfechos. Tal intervenção possibilitaria, além da redução de morbidade, a redução da necessidade de serviços ambulatoriais e por vezes hospitalares.

Não foram, todavia, encontradas evidências de benefício relevante da antibioticoprofilaxia quando foram analisados apenas ensaios que incluíram mulheres em cesáreas eletivas. A razão de taxas para a endometrite foi bastante favorável à intervenção, mas com intervalo amplo, próximo a um, sendo, porém pouco relevante a diferença de taxa encontrada e sem significância estatística. A principal limitação dessa análise foi o número reduzido de ensaios, o que não nos permite concluir em relação ao efeito da profilaxia nesse grupo de mulheres.

Para as cesáreas não eletivas, apesar das variações no tempo de exposição à ruptura de membrana e também nas medidas dos desfechos, houve consistência nos achados nesse grupo de mulheres: todos os estudos obtiveram resultados claramente favoráveis à antibioticopro- 
filaxia, havendo, portanto, evidência forte de que antibioticoprofilaxia reduz de forma substancial os percentuais de infecções pós-cirúrgicas, principalmente, a endometrite.

Apenas três ensaios selecionados compararam esquemas de dose única e múltiplas doses, havendo ainda questões quanto ao prognóstico das pacientes, a exclusões e relato de perdas e forma de análise dos dados, o que limitou bas-

\section{Resumo}

O objetivo do trabalho foi analisar a evidência científica disponivel sobre os efeitos da antibioticoprofilaxia em cesarianas. As presentes revisões sistemáticas compreenderam um exame detalhado da qualidade do desenho e da execução assim como da heterogeneidade clínica entre os ensaios. A meta-análise dos ensaios placebo-controlados (27 estudos) apontou eficácia aproximada de $65 \%$ para ambos os desfechos estudados, endometrite e infecção da ferida cirúrgica (IFC), correspondendo a uma queda da incidência de $11 \%$ e 5\%, respectivamente. As análises de sensibilidade mostraram efeitos sumários semelhantes aos observados para o conjunto dos ensaios. O subgrupo dos 12 ensaios de cesáreas não eletivas indicou benefício importante da antibioticoprofilaxia para ambos os desfechos, correspondente a uma queda de $14 \%$ (endometrite) e 5\% (IFC). Para cesáreas eletivas (dois ensaios), não foi mostrado benefício relevante. A evidência obtida dos ensaios comparativos de doses foi limitada devido a falhas metodológicas importantes e ao pequeno número de pacientes envolvidas (três ensaios). Os ensaios comparativos de antimicrobianos (sete estudos) não evidenciaram diferença de eficácia entre os dois esquemas analisados, cefalosporinas de $1 \underline{a}$ e de $2 \underline{a}$ geração.

Cesárea; Antibioticoprofilaxia; Literatura de Revisão tante a referida análise. Devido à quantidade de pacientes estudadas e às limitações dos ensaios selecionados, a nossa meta-análise não pode concluir em relação à comparação dos regimes de dose única e múltiplas doses.

Finalmente, em relação aos ensaios comparativos de tipos de antimicrobianos não foi evidenciada diferença entre os dois esquemas analisados, cefalosporinas de 1ạ e de 2ạ geração.

\section{Colaboradores}

A. C. M. Martins e L. Krauss-Silva participaram de todas as etapas de identificação de estudos, coleta, análise e síntese dos dados, estruturação e redação do texto. 


\section{Referências}

1. Goldenberg RL, Andrews WW, Hauth JC. Choriodecidual infection and preterm birth. Nutr Rev 2002; 60:S19-25.

2. Yudin M, Landers V, Meyn L, Hillier S. Clinical and cervical cytokine response to treatment with oral or vaginal metronidazole for bacterial vaginosis during pregnancy: a randomized trial. Obstet Gynecol 2003; 102:527-34

3. Andrews WW, Sibai BM, Thom EA, Dudley D, Ernest JM, McNellis D, et al. Randomized clinical trial of metronidazole plus erythromycin to prevent spontaneous preterm delivery in fetal fibronectinpositive women. Obstet Gynecol 2003; 101:847-55.

4. Klebanoff MA, Schwebke JR, Zhang J, Nansel TR, Yu KF, Andrews WW. Vulvovaginal symptoms in women with bacterial vaginosis. Obstet Gynecol 2004; 104:267-72.

5. Henderson E, Love EJ. Incidence of hospital-acquired infections associated with caesarean section. J Hosp Infect 1995; 29:245-55.

6. Ministério da Saúde. Consenso sobre uso racional de antimicrobianos. Brasília: Ministério da Saúde; 1998.

7. Altman D. Practical statistics for medical research London: Chapmam \& Hall; 1999.

8. Burke JF. The effective period of preventive antibiotic action in experimental and dermal lesions. Surgery 1961; 50:161-8.

9. Shapiro M, Shimon D, Freund U, Sacks T. A decisive period in the antibiotic prophylaxis of cutaneous lesions. J Infect Dis 1980; 141:532.

10. Classen DC, Evans RS, Burke JP. The timing of prophylaxis administration of antibiotics and the risk of surgical - wound infection. N Engl J Med 1992; 326:281-6.

11. Stone HH, Hooper CA, Kolb LD, Geheber CE Dawkins EJ. Antibiotic prophylaxis in gastric, biliar and colonic surgery. Ann Surg 1976; 184:443-52.

12. Atkinson MW, Owen J, Wren A, Hauth JC. The effect of manual removal of the placenta on post-cesarean endometritis. Obstet Gynecol 1996; 87:99-102.

13. Ehrenkranz NJ, Blackwelder WC, Pfaff SJ, Poppe D, Yerg DE, Kaslow RA. Infections complicating lowrisk cesarean sections in community hospitals: efficacy of antimicrobial prophylaxis. Am J Obstet Gynecol 1990; 162:337-43.

14. Watts DH, Hillier SL, Eschenbach DA. Bacterial vaginosis as a risk factor for post-cesarean endometritis. Obstet Gynecol 1990; 75:52-8.

15. Smaill F, Hofmeyr GJ. Antibiotic prophylaxis for cesarean section (Cochrane Review). In: The Cochrane Library, Issue 2, 2004. Oxford: Update Software.

16. Chelmow D, Ruehli MS, Huang E. Prophylactic use of antibiotics for nonlaboring patients undergoing cesarean delivery with intact membranes: a metaanalysis. Am J Obstet Gynecol 2001; 184:656-61.

17. Hopkins L, Smaill F. Antibiotic prophylaxis regimens and drugs for cesarean section (Cochrane Review). In: The Cochrane Library, Issue 2, 2004. Oxford: Update Software.

18. Sacks HS, Chalmers TC, Reitman D, Berk A. Metaanalysis of randomized controlled trials. $\mathrm{N}$ Engl J Med 1987; 316:450-5.
19. Oxman AD. Checklists for review articles. BMJ 1994; 309:48-51.

20. Thompson SG. Why sources heterogeneity in meta-analysis should be investigated. BMJ 1994; 309:1351-5.

21. Centers for Disease Control and Prevention. CDC definitions of nosocomial surgical site infections, 1992: a modification of CDC definitions of surgical wound infections. Infect Control Hosp Epidemiol 1992; 13:608-8.

22. Centers for Disease Control and Prevention Guideline for prevention of surgical site infection. Atlanta: Centers for Disease Control and Prevention; 1999.

23. Young R, Platt L, Ledger W. Prophylactic cefoxitin in cesarean section. Surg Gynecol Obstet 1983; 157:11-4.

24. Duff P, Park RC. Antibiotic prophylaxis for cesarean section in a military population. Mil Med 1980 145:377-81.

25. Gibbs RS, De Cherney AH, Schwarz RH. Prophylactic antibiotics in cesarean section: a double-blind study. Am J Obstet Gynecol 1972; 114:1048-53.

26. Roex AJM, Puyenbroek JI, MacLaren DM, van Geijn HP, Arts NFT. A randomized clinical trial of antibiotic prophylaxis in cesarean section: maternal morbidity, risk factors and bacteriological changes. Eur J Obstet Gynecol Reprod Biol 1986; 22:117-24.

27. Hawrylyshyn PA, Bernstein EP, Papsin FR. Shortterm antibiotic prophylaxis in high-risk patients following cesarean section. Am J Obstet Gynecol 1983; 145:285-9.

28. Gall S, Hill G. Single-dose versus multiple-dose piperacillin prophylaxis in primary cesarean operation. Am J Obstet Gynecol 1987; 157:502-6.

29. Kreutner AK, Del Bene VE, Delamar D, Bodden JL, Loadholt CB. Perioperative cephalosporin prophylaxis in cesarean section: effect on endometritis in the high-risk patient. Am J Obstet Gynecol 1979; 134:925-35.

30. Hager WD, Rapp RP, Billeter M, Bradley BB. Choice of antibiotic in nonelective cesarean section. Antimicrob Agents Chemother 1991; 35:1782-4.

31. Reid G, Bocking A. The potential for probiotics to prevent bacterial vaginosis and preterm labor. Am J Obstet Gynecol 2003; 189:1202-8.

32. Duff P, Smith PN, Keiser JF. Antibiotic prophylaxis in low-risk cesarean section. J Reprod Med 1982; 27:133-8.

33. Bagratee JS, Moodley J, Kleinschmidt I, Zawilski W. A randomised controlled trial of antibiotic prophylaxis in elective caesarean delivery. BJOG 2001; 108:143-8.

34. Wong R, Gee CL, Ledger WJ. Prophylactic use of cefazolin in monitored obstetric patients undergoing cesarean section. Obstet Gynecol 1978; 51: 407-11.

35. Rehu M, Jahkola M. Prophylactic antibiotics in Caesarean section: effect of a short preoperative course of benzyl penicillin or clindamycin plus gentamicin on postoperative infectious morbidity. Ann Clin Res 1980; 12:45-8. 
36. Gibbs RS, St. Clair PJ, Castillo MS, Castaneda YS. Bacteriologic effects of antibiotic prophylaxis in high-risk cesarean section. Obstet Gynecol 1981; 57:277-82.

37. Harger JH, English DH. Selection of patients for antibiotic prophylaxis in cesarean sections. Am J Obstet Gynecol 1981; 141:752-8.

38. Stage AH, Glover DD, Vaughan JE. Low-dose cephradine prophylaxis in obstetric and gynecologic surgery. J Reprod Med 1982; 27:113-9.

39. Stiver HG, Forward K, Livingstone R, Fugere P, LeMay M, Verschelden G, et al. Muliticenter comparison of cefoxitin versus cefazolin for prevention of infectious morbidity after nonelective cesarean section. Obstet Gynecol 1983; 143:158-63.

40. Fugere P, Turgeon P, Boucher M, Verscheiden G, Lemay M. Utilisation des cephalosporines comme antibioprophylaxie lors de cesariennes. Can Med Assoc J 1983; 129:132-5.

41. Saltzman DH, Eron LJ, Kay HH, Sites JG. Singledose antibiotic prophylaxis in high-risk patients undergoing cesarean section. Obstet Gynecol 1985; 65:655-7.

42. Hagglund L, Christensen KK, Christensen P, Westrom L, Ingemarsson I. Reduced rate of postoperative infections in emergency cesarean section after two doses of cefuroxim perioperatively. Acta Obstet Gynecol Scand 1989; 68:201-4.

43. Ruiz-Moreno JA, García-Rojas JM, Lozada-León JD. Prevention of post cesarean infectious morbidity with a single dose of intravenous metronidazole. Int J Gynecol Obstet 1991; 34:217-20.

44. Ismail MA, Nelson KE, Larson P, Moses VK. Selective effect of cefoxitin prophylaxis on post-cesarean-section microbial flora. J Reprod Med 1990; 35:168-74.

45. Duff P, Robertson A, Read J. Single-dose cefazolin versus cefonicid for antibiotic prophylaxis in cesarean delivery. Obstet Gynecol 1987; 70: 718-21.

46. Carlson C, Duff P. Antibiotic prophylaxis for cesarean delivery: is an extended-spectrum agent necessary? Obstet Gynecol 1990; 76:343-6.
47. Peterson CM, Medchill M, Gordon D, Chard H. Cesarean prophylaxis: a comparison of cefamandole and cefazolin by both intravenous and lavage routes, and risk factors associated with endometritis. Obstet Gynecol 1990; 75:179-82.

48. Gibbs RS, Hunt JE, Schwarz RH. A follow-up study on prophylactic antibiotics in cesarean section. Am J Obstet Gynecol 1973; 117:419-22.

49. Dillon WP, Seigel MS, Lele AS, O'Leary JA. Evaluation of cefoxitin prophylaxis for cesarean section. Int J Gynaecol Obstet 1981; 19:133-9.

50. Polk BF, Krache M, Phillippe M, Muñoz A, Hutchinson D, Miao L, et al. Randomized clinical trial of perioperative cefoxitin in preventing maternal infection after primary cesarean section. Am J Obstet Gynecol 1982; 142:983-7.

51. Hager WD, Williamson MM. Effects of antibiotic prophylaxis on women undergoing nonelective cesarean section in a community hospital. J Reprod Med 1983; 28:687-90.

52. Padilla SL, Spence MR, Beauchamp PJ. Single-dose ampicillin for cesarean section prophylaxis. Obstet Gynecol 1983; 61:463-6.

53. Tully L, Gates S, Brocklehurst P, McKenzie-McHarg $\mathrm{K}$, Ayers S. Surgical techniques used during caesarean section operations: results of a national survey of practice in the UK. Eur J Obstet Gynecol Reprod Biol 2002; 102:120-6.

54. Karhunen M, Koskela O, Teisala K, Suikkari AM, Mattila J. Prophylaxis and treatment of anaerobic infections following caesarean section with tinidazole. Chemotherapy 1985; 31:228-36.

55. Racinet C, Mallaret MR, Favier M, Berthet J, Morel I, Fauconnier J, et al. Antibiotic prophylaxis in cesarean sections without high risk of infection. Presse Med 1990; 19:1755-8.

56. Yip SK, Lau TK, Rogers MS. A study on prophylactic antibiotics in cesarean sections - is it worthwhile? Acta Obstet Gynecol Scand 1997; 76:547-9.

Recebido em 27/Out/2005

Versão final reapresentada em 26/Mai/2006

Aprovado em 12/Jun/2006 